\title{
BARTER ECONOMIES AND CENTRALIZED MERCHANTS
}

\author{
José Noguera \\ CERGE-EI, Charles University \\ P.O. Box 882, Politických vězňů 7, 11121 Prague 1 \\ Phone: 420-2-240.05.107 \\ jose.noguera@cerge.cuni.cz
}

\begin{abstract}
$\underline{\text { Abstract }}$
This essay analyzes the circumstances under which a barter system based on merchants and centralized markets dominates an unstructured primitive barter system. For that purpose, we set up a spatial general equilibrium model where exchange is costly. In an unstructured barter system, as population increases, the transaction costs may become cumbersome and the cost of trade expansion surpasses its benefits. This imposes limitations on the scope of the economy and the production level. To overcome these limitations, rational individuals can develop a more advanced barter system based on centralized merchants. This may explains why some economies, like the ancient Egypt and the Incas in Peru, did not evolve to a monetary system, and kept barter as their main exchange system.
\end{abstract}

JEL: E0, R10, R11

Key Words: Primitive Barter, Redistributive Barter, Merchants, Spatial General Equilibrium 


\section{BARTER ECONOMIES AND CENTRALIZED MERCHANTS}

\section{INTRODUCTION}

The understanding of barter economies is a very important matter in economic theory. Although we are sometimes tempted to study them in the context of modern economies, doubtless the natural starting point for studying barter economies are ancient and primitive societies. In the distant past our ancestors lived in a moneyless, primitive communal system in which people produced or found everything they needed to live on, and there were no organized markets. Einzig (1966, pg. 333) summarizes the conditions under which a moneyless primitive communal system existed. They encompass low levels of intelligence, absence of sense of values, low economic development, the absence of private property, no moral or religious taboos, and a scarcity or distrust of a good that could serve well as money. However, once these conditions were overcome, barter economies appeared and in time, converged to some kind of monetary economy.

Hicks (1969) argues that before the appearance of the division of labor and a systematic trading system, societies already had some type of organization. Such an organization requires the existence of cities where people interrelate. An organization like this precedes the emergence of specialized producers. After this initial stage of primitive trade, Polanyi (1957) finds economies moving on to economic systems with a more complete division of labor where people exchange their surpluses. He argues that a Redistributive Barter System, a system in which goods are sent from the production place to a market center and then redistributed, dominates nonmonetary economies. In the redistributive barter system, individuals produce one or a few goods and consume many. They usually go to a market center, often located at the center of the city, and 
trade the only commodity they have for all other commodities they need. Such trade was made through merchants, which dominate the early trade based on pure barter. Polanyi argues that, although sometimes we find peddler merchants in such a system traveling from city to city, exchanging different kinds of goods and playing an important role for facilitating trade between market centers, their role was less important in the direct exchange with final consumers. Most anthropologists, see for example Polanyi (1957), Einzig (1966), Davies (1995), and Renfrew and Bahn (1996), agree that the redistributive barter system emerged out of a primitive barter system and that, with all likelihood, money appeared under this system. Economists like Ederer (1964), Hicks (1969, 1989), Clower (1995), and Clower and Howitt (2000) also highlight the role of centralized merchants as a previous stage to a monetary system.

Two well-known examples of redistributive barter systems are the ancient Egypt and the Incas in Peru. In the first case, a medium of exchange appeared during the intermediate period after the Old Kingdom [Romer (1990)]. The latter never adopted a medium of exchange [Brundage (1985)]. One important feature of these two economies was the presence of a strong central authority that controlled and organized the intermediation activity [Renfrew and Bahn (1996), pp. 351-353].

Based on these ideas, this essay analyzes the circumstances under which a redistributive barter system with centralized merchants dominates over a primitive barter system. For that purpose, we write a spatial general equilibrium model where exchange is costly to compare a primitive unstructured barter economy with a redistributive barter economy.

Section II shows a model of an unstructured barter economy where individuals want to consume all goods available. Every individual, who is a specialized producer, meets and barters with every other individual in the central market to acquire the consumption goods they need. To trade successfully, they incur some transaction costs, which include transport and barter costs. 
Section III extends the model to incorporate intermediaries. Barter activity is done through centralized merchants allowing producers to increase their production. Every producer, however, must give up a share of his production to pay for intermediation services.

Based on the models developed in Sections II and III, Section IV determines the conditions under which the redistributive barter economy with centralized merchants dominates over the primitive unstructured barter economy. We prove that after population reaches a critical size, the emergence of intermediaries contributes to economic growth by lowering the unit cost of bartering and inducing the creation of new goods; then, we adopt ad hoc dynamics to provide an explanation to the anthropological evidence that motivates this article. Section V concludes.

\section{THE PRIMITIVE BARTER ECONOMY}

\section{Assumptions of the Model}

Consider a narrow city developed on a line along a river that flows from location zero to location $G_{p}$, where a continuum of $L$ individuals lives. At every location, only one individual locates, and each of them is simultaneously a producer and a consumer. Every individual specializes in producing only one good, so individuals can be identified by their location, and also the good that they produce. They operate in a spatial monopolistic competitive market, so they maximize price and there is free entry.

An undesirable feature frequently present in spatial monopolistic competitive models is that individuals' income varies across locations [see Fujita et. al (1999, Ch. 4) for a discussion]. To avoid the complications of dealing with unequals, and to keep the model mathematically tractable, we introduce land and a redistributive government, which owns the land for the city; then, the government leases the land to individuals at the competitively determined rent at each location $i$, and distributes the revenue from land equally among producers. 
Each individual sends her production to a market center created at location zero to barter it by the goods that she needs for consumption. Thus, as suggested by Hicks (1969), the environment is an organized society: there is a city, a government, and a central place where individuals interrelate and barter their surpluses. There are no intermediaries and no one can transport goods upstream. There is free mobility of factors and everyone consumes at the market center. We refer to this economy as the Primitive Barter Economy (PBE). Notice that, in the PBE, the number of goods is equal to the number of individuals, that is, $L=G_{p}$.

\section{Consumers' Behavior}

There are to be assumed that all goods enter symmetrically into individuals' demand. The consumer that lives at location $i$ has the following CES utility function

$$
U^{i}=\left(\int_{0}^{G_{p}}\left(c_{j}^{i}\right)^{p} d j\right)^{1 / \rho}
$$

where $c_{j}^{i}$ is individual $i$ 's consumption of $\operatorname{good} j$, and $\rho$ is a constant between zero and one that represents the intensity of the preference for variety. Therefore, $\sigma=1 /(1-\rho)$ is the elasticity of substitution between any two goods, and it is constant and greater than one. As $\rho$ decreases, the desire to consume a greater variety of goods increases, and the elasticity of substitution between any two goods decreases.

Let $P_{j}$ be the delivered or c.i.f. price of $\operatorname{good} j$, and $Y_{i}$ the net income of the individual dwelling at $i$; then, individual $i$ faces the budget constraint $Y_{i} \geq \int_{0}^{G_{p}} P_{j} c_{j}^{i} d j$. As usual in DixitStiglitz type models, the consumer price index is

$$
H=\left(\int_{0}^{G_{p}} P_{j}^{1-\sigma} d j\right)^{1 /(1-\sigma)}
$$


and individual $i$ 's consumption function for every variety $j \in\left[0, G_{p}\right]$ is

$$
c_{j}^{i}=Y P_{j}^{-\sigma} H^{\sigma-1} .
$$

\section{Producers' Behavior}

The individual dwelling at location $j$ away from the market center produces $x_{j}$ units of good $j$; then, he ships the output to the market center, incurring in some transport costs. To keep the things simple, assume that these costs take the Samuelson (1952) and von Thünen (1826)'s iceberg form, that is, if a unit of good $i$ is dispatched from location $i$ to the market center, a fraction $\tau i$ melts away in transit, $0<\tau<1$.

Once in the market center, individual $j$ quotes her merchandize at a price $P_{j}$ and spends some time meeting all other individuals to barter the only good that she has for all other goods that she needs. Producers' only input is labor, measured in units of time. Thus, the primitive barter technology represents an opportunity cost for every individual. Assume that if an individual must barter with $G_{p}$ different individuals, their opportunity cost is a share $\gamma G_{p}$ of its potential production, $x_{i}$. Therefore, as the scope of the economy $\left(G_{p}\right)$ increases, individuals spend more resources to succeed bartering. Notice that if individual $j$ wants to consume the good produced by individual $k$, then $j$ wants to consume only some of $k$ 's production but not her entire production. Therefore, the coincidence of wants is not complete and $j$ must trade the rest of her production with other individuals until she acquires all the goods that she needs.

The producer located at $j$ incurs the following labor cost: $l_{j}=a+b x_{j}$, where $a, b>0$. Assume that the producer acts as a monopolistic competitive firm, and let $W_{j}$ be her unit labor remuneration. Then, considering the barter and transport costs, if the potential production of the individual located at $j$ is one unit of $\operatorname{good} j$, she will barter only $(1-\tau j-\gamma G)$ in the market center. Thus, the firm's profit function is 


$$
\pi_{j}=\left(1-\tau j-\gamma G_{p}\right) P_{j} x_{j}-W_{j}\left(a+b x_{j}\right)
$$

Since every individual is simultaneously the firm's owner and the only worker, there must be zero profit, and the labor remuneration is the break-even wage at $j$. In other words, the individual located at $j$ works $l_{j}$ units of time to produce $x_{j}$ units of good $j$. This merchandize has a value equal to $\left(1-\tau j-\gamma G_{p}\right) P_{j} x_{j}$ at the market center, which at the same time is equal to her labor remuneration. Consider the equilibrium condition for $\operatorname{good} j \in\left[0, G_{p}\right]$,

$$
\left(1-\tau j-\gamma G_{p}\right) x_{j}=\int_{0}^{L} c_{j}^{i} d i,
$$

and suppose that the price of one good has no effect on the demand for any other good; then, the profit-maximizing price for the producer located at $j \in\left[0, G_{p}\right]$ is

$$
P_{j}=\frac{b W_{j}}{\rho\left(1-\tau j-\gamma G_{p}\right)} .
$$

Hence, producer $j$ sets a higher price for $\operatorname{good} j$ the farther away the production site is, and the more expensive the barter system is. The zero profit condition allows finding individual's potential output, $x_{j}=a(\sigma-1) / b$. Since $a, b$ and $\sigma$ are the same for all firms, we can use the shorthand $x$ to refer to any individual's output; then, every individual's labor is $l=a \sigma$. Since we can freely choose the units of measurement for labor, we set the fixed input requirement to satisfy $a \sigma=1$, so every individual spends one unit of labor in production, that is, $l=1$. We regard this as the individual's labor endowment.

The individual residing at location $j$ obtains an income is $Y_{j}=W_{j}-R(j)+T D R / L$, where $R(i)$ is the market rent and TDR is the total differential rent equally distributed by the government among producers. The free mobility assumption implies that the market rent adjusts to equalize individuals' equilibrium utility. Thus, individuals' equilibrium income must be the same in all locations. This allows dropping the subscript in individuals' income and denoting it simply by $Y$. 


\section{City Growth}

We can now proceed to analyze how the economy behaves as the city grows, that is, as population increases. Since all producers have the same equilibrium income, every individual consumes $\left(1-\tau j-\gamma G_{p}\right) x / L$ units of good $j$. Considering that $L=G_{p}$ in the PBE, after doing some computations we can write the utility function as

$$
U_{p b e}=\frac{A_{0}}{L}\left[(1-\gamma L)^{2-1 / \sigma}-(1-(\tau+\gamma) L)^{2-1 / \sigma}\right]^{\sigma /(\sigma-1)} .
$$

Theorem 1: The scope of the economy $\left(G_{p}\right)$ and the city population $(L)$ are bounded by $1 /(\tau+\gamma)$.

The result arises because it is impossible to produce some goods at those peripheral locations beyond $1 /(\tau+\gamma)$, since no time would be leftover to individuals after transport and barter costs are netted out of the total labor endowment. Hence, the economy cannot be so large and the city cannot be so long. Notice also that $U_{p b e}$ is only well defined for $0<L<1 /(\tau+\gamma)$.

Theorem 2: The equilibrium utility function in the PBE has the following properties:

i) $\quad U_{p b e}$ tends to zero as population $(L)$ tends to zero;

ii) $U_{p b e}$ tends to $A_{0} \tau^{(2 \sigma-1) /(\sigma-1)}(\tau+\gamma)^{-\sigma /(\sigma-1)}$ as $L$ tends to the city population upper bound;

iii) As $L$ tends to zero, the slope of $U_{p b e}$ tends to zero if $\sigma<2$, tends to infinity if $\sigma>2$, and tends to $\tau(2 \sigma-1) / \sigma$ if $\sigma=2$;

iv) $U_{p b e}$ reaches a maximum at $L_{p}$, where $0<L_{p}<1 /(\tau+\gamma)$.

Proof: see Appendix A.

Figure 1 shows the equilibrium utility function in the PBE in the case where $\sigma>2$. If $\sigma$ were less than 2 , the only difference is that the curve is convex for small populations. 
Observe some interesting properties of the PBE. First, in her shopping trip, every individual meets every other individual at the market center. However, she can only barter a share of her production, and therefore, there is still a lack of a double coincidence of wants problem.

Second, the number of goods produces satisfaction, and individuals always obtain benefits from agglomeration. Taste for variety makes the consumer choose a quantity of all new goods appearing in the market, regardless of their price. Even if the city population grows beyond $L_{p}$, where individuals' utility in the PBE reaches a maximum, there is no incentive to live isolated since in this case individuals' utility would be zero.

Third, growth in the PBE has a limitation since the scope of the economy is bounded. In fact, if population becomes greater than or equal to $1 /(\tau+\gamma)$, individuals dwelling at that distance or farther spend much time bartering and traveling to the market center. The combined cost of both activities would leave the individual with no output to barter at the city center.

\section{THE BARTER ECONOMY WITH MERCHANTS}

Since bartering is costly, it may occur to someone to accept producer's output, barter it for all the goods he desires, and charge a fee for her service [Hicks (1989) and Clower (1995)]. If this fee represents a lower barter cost, the producer would be better off if she dealt with an intermediary, and a Redistributive Barter Economy with Merchants emerges. In this section, we extend the model to allow the presence of intermediaries.

\section{Assumptions of the Model}

Suppose that there are $F$ individuals playing the role of centralized merchants in the economy. They produce nothing but devote their time facilitating exchanges. Any individual can meet a centralized merchant and barter all the goods that she brings for all other goods she wants, that is, for a bundle containing some of each of the $G_{b}$ goods existing in this economy. Producer $j$ quotes 
his output at a price $P_{j}$, and the merchant charges a markup $\xi$ for its intermediation service, so consumers pay a price $\xi P_{j}$ for one unit of $\operatorname{good} j$.

Since each centralized merchant barters a bundle containing all goods for a quantity of only one good with the producer, each merchant obtains directly from producers, at most, $2 g / F$ goods, so each merchant must barter with other merchants to purvey her storage. Suppose that the Government organizes the central market in such a way that merchants can exchange the merchandize among them at an insignificant cost.

Producers are still located away and go the market center for barter purposes. Yet, they now physically meet only one centralized merchant and not all other individuals, so they substantially reduce their barter cost. Assume, for simplicity, that this cost is insignificant. Producers behave in a spatial monopolistic competitive environment as defined for the PBE. Assume also a full employment condition, so $L=G_{b}+F$. Notice that now $L$ is greater than $G_{b}$. We refer to this economy as the Redistributive Barter Economy (RBE).

\section{Consumers and Producers' Behavior}

Consumers and producers' behavior is similar in both the RBE and the PBE. However, there are two important differences. First, in the RBE, producers do not bear the troublesome barter cost of meeting many other producers; and second, since consumers must pay the merchant's markup, they now pay $\xi P_{j}$ to acquire one unit of consumption good $j$. This causes some small modifications in consumers and producers' behavior. First, the consumer price index becomes

$$
H=\left(\int_{0}^{G_{b}}\left(\xi P_{j}\right)^{1-\sigma} d j\right)^{1 /(1-\sigma)}
$$

and the consumption function of $\operatorname{good} j \in[0, G]$ for individual $i, c_{j}=Y_{i} \xi^{-\sigma} P_{j}^{-\sigma} H^{\sigma-1}$. Producer $j$ now exchanges $(1-\tau j) x_{j}$ of output, equilibrium condition of good $j \in\left[0, G_{b}\right]$ changes to 
$(1-\tau j) x_{j}=\int_{0}^{L} c_{j}^{i} d i$, and the profit-maximizing price to $P_{j}=\frac{b W_{j}}{\rho(1-\tau j)}$. The free mobility assumption guarantees that each producer obtains the same income, $Y$.

\section{The Intermediaries}

Each centralized merchant prepares a bundle containing $c_{j}$ units of good $j \in\left[0, G_{b}\right]$ to barter it with individuals. Since all merchants are identical, we may consider symmetric equilibrium. Labor is the only merchant's cost, and every centralized merchant charges a markup $\xi$. Thus, the merchant's income is

$$
(\xi-1)\left(c_{m}+\int_{0}^{G_{b}} P_{j} c_{j} d j\right) L / F
$$

Using individuals' budget constraint, we can write this expression as $(1-1 / \xi) Y L / F$. The free entry condition guarantees that each centralized merchant obtains the same income as producers, $Y$. This allows obtaining the merchant's markup $\xi=L /(L-F)$, and from the full employment condition, we have $\xi=L / G_{b}$, that is, the merchant's markup is the ratio between population and the number of producers.

Assume that the intermediation activity works with a constant return to scale technology, that is, $F$ workers can barter up to $K F$ units of manufactured goods, regardless of their variety. Since the whole production is bartered through intermediaries,

$$
F K=x \int_{0}^{G_{b}}(1-\tau j) d j
$$

Considering this and the full employment condition, we find the equilibrium number merchants and producers

$$
G_{b}(L)=\eta-\sqrt{\eta^{2}-\frac{2}{\tau} k L} \quad \text { and } \quad F=L-\eta+\sqrt{\eta^{2}-\frac{2 k}{\tau} L}
$$


where $k=K / x$ is the ratio between the merchant's capacity to intermediate $(K)$ and the producer's potential output $(x)$, and $\eta=(1+k) / \tau$. Notice that $F$ is always positive. In fact, $F>0$ if and only if $k>1 / 2$, i.e., $K>x / 2$. Yet, if an individual owns the technology to produce and barter $x$ units of some good, a merchant must be able to barter at least the same amount of merchandize. Therefore, $k>1$ and this condition always holds.

Taking derivatives with respect to $L$, it can be checked that $G_{b}{ }^{\prime}, G_{b}{ }^{\prime}$ ' and $F_{b}$ ' are positive, and $F_{b}{ }^{\prime \prime}$ is negative. In addition, with a bit of calculus, we can prove that the number of merchants increases and the number of producers decreases, as transport cost $(\tau)$ increases or the merchant capacity decreases $(K)$. This reflects the cost of transport and barter activities in the production of goods and the intermediation services.

\section{City Growth and Transport Costs}

Let's proceed to analyze the economy's behavior as the city grows. Since all individuals obtain the same equilibrium income, each of them consumes $(1-\tau j) x / L$ units of $\operatorname{good} j \in\left[0, G_{b}\right]$. Thus, after using some calculus, we can write equilibrium utility as

$$
U_{r b e}=\frac{A_{0}}{L}\left[1-(1-\tau G(L))^{2-1 / \sigma}\right]^{\sigma /(\sigma-1)} .
$$

Theorem 3: In the RBE, the scope of the economy $\left(G_{b}\right)$ is bounded by $1 / \tau$, and population cannot grow to more than

$$
L_{\max }=\frac{1}{\tau}\left(1+\frac{1}{2 k}\right) .
$$

Since producers cannot bring their production from those peripheral locations farther than $1 / \tau$; then, from (2), we obtain $L_{\max }$. This imposes a limitation on the RBE. Notice also that the utility function in the RBE is only well defined for $0<G(L)<1 / \tau$.

Theorem 4: The equilibrium utility function in the RBE has the following features: 
i) $U_{r b e}$ tends to zero as $L$ tends to zero;

ii) $U_{r b e}$ tends to $\frac{2 k \tau A_{0}}{2 k+1}$ as $L$ tends to the maximum possible population in the RBE, $L_{\max }$;

iii) $U_{\text {rbe }}$ tends to $A_{0}(\gamma+\tau)\left[1-\left(\sqrt{1+k^{2}+2 k-2 k \frac{\tau}{\gamma+\tau}}-k\right)^{2-1 / \sigma}\right]^{\sigma /(\sigma-1)}$ as $L$ tends to the maximum possible population in the $\mathrm{PBE}, 1 /(\gamma+\tau)$;

iv) $U_{r b e}$ is an increasing function with respect to population.

Proof: see Appendix B.

\section{THE PBE AND THE RBE COMPARED}

It is important to note several facts in comparing the PBE and the RBE. First, the presence of the centralized merchants allow overcoming the inconvenience of the unstructured barter system without adopting a monetary system, since the producer already knows that a merchant will barter his entire production for the consumption goods that she needs.

Second, in the RBE, producers go to the market center only to meet a merchant and not all other individuals. This allows them to increase their production and barter a bigger amount of output for other goods. On the other hand, they give up a share of their production to the merchants.

Third, prices reflect the transport cost identically in both the PBE and the RBE. However, barter costs are reflected different in both systems. In the PBE, it is reflected by a lower production available for bartering that diminishes the supply and increases the price. In the RBE, the producer's price is lower, and the markup reflects the fact that the producer gives up a share of his production to pay for the intermediation services provided by the merchants.

Fourth, consider the difference $\Delta U=U_{r b e}-U_{p b e}$. If $\Delta U$ is positive, the RBE dominates over the PBE. If not, the PBE dominates and no merchant is around. Let's inquire the 
circumstances under which the RBE dominates over the PBE. Notice that the PBE always dominates the RBE for small economies. In fact, taking the limit of $U_{r b e} / U_{p b e}$ as $L$ tends to zero, we have

$$
\lim _{L \rightarrow 0} \frac{U_{r b e}}{U_{p b e}}=\left[\frac{k}{k+1}\right]^{\sigma /(\sigma-1)}
$$

which is always less than one. The reason is that a small population does not allow intermediaries to take advantage of the economies of scale.

Given the geometrical properties of $U_{p b e}$ and $U_{r b e}$, we may consider only three possible cases. First, the PBE always dominates the RBE for any population level (Figure 2). Second, the RBE dominates the PBE after it reaches a maximum (Figure 3), and third, the RBE dominates the PBE before it reaches a maximum (Figure 4).

\section{FIGURE 2, 3 AND 4 COME HERE}

If the RBE dominates over the PBE at some point, it must occur that $U_{r b e}>U_{p b e}$ at the city's lowest upper bound population in the PBE, that is, at $L=1 /(\tau+\gamma)$. From Theorems 2 and 4 , this occurs if

$$
J(\tau, \gamma, \sigma)+F(k, \tau, \gamma, \sigma)<1
$$

where $J(\tau, \gamma, \sigma)=\left(\frac{1}{1+\gamma / \tau}\right)^{2-1 / \sigma}$ and $F(k, \tau, \gamma, \sigma)=\left(\sqrt{(1+k)^{2}-\frac{2 k}{1+\gamma / \tau}}-k\right)^{2-1 / \sigma}$. Let's analyze the conditions in which (4) holds. This inequality is affected by four parameters: $\sigma, k$, $\tau$ and $\gamma$. $J(\sigma, \tau, \gamma)+F(\sigma, k, \tau, \gamma)$ is a decreasing function with respect to both $\sigma$ and $k$. Consider first the elasticity of substitution $(\sigma)$. If the intensity of the preference for variety is too strong, for example $\rho$ tends to zero, so $\sigma$ tends to one, individuals always prefer to consume more variety to more quantity, and inequality (4) reverses. In this case, the PBE never dominates the RBE, and 
no merchant ever appears. This also means that, although taste for variety plays an important role in our story, it cannot be as high as to make individuals always prefer variety to quantity.

Consider now $k$. An increase in $k$ means that the intermediation technology increases relative to producers', so the benefits from the economies of scale in intermediation increase. Thus, a higher $k$ encourages the emergence of a RBE.

Assume that modern individuals have the same preference for variety as our ancestors, so we can follow Fujita, Krugman and Venables (1999) and choose $\sigma=5$ in the rest of the analysis. Suppose that $k=1$. For these parameter values, using numerical calculus we find that inequality (4) holds if $\gamma>0.27 \tau$, that is, if the opportunity cost of bartering $(\gamma)$ is at least $27 \%$ of the transport cost of shipping the merchandize one unit of distance $(\tau)$. Now, as it was argued before, even in the most primitive economy, $k$ is much higher than one. Suppose that the intermediation technology increases to, say $k=2$, then inequality (4) holds if $\gamma>0.07 \tau$; and for $k=6$, it holds if $\gamma>0.01 \tau$. This means that any major advance in the transport technology, say the invention of the wheel and wheelbarrows, could cause the emergence of intermediaries.

Let's analyze this result closer. First, the advantage of using intermediaries strongly increases as $k$ increases. Second, if the transport cost is so high that population in the PBE never reaches the necessary size, individuals will be better off if they do not deal with an intermediary and a primitive unstructured barter prevails. Yet, once the transport technology reaches the minimum necessary level the necessary conditions appear and make inequality (4) hold.

Third, the higher the opportunity cost of bartering $(\gamma)$, the lower the necessary population to take advantages of the economies of scale in intermediation is. We may think that the cost of exchanging $(\gamma)$ increases with population. In fact, the idea that the inconveniences of the barter system are caused by the absence of a double coincidence of wants problem has been around at least since Adam Smith, and has been used to justify the existence of a medium of exchange. The 
modern literature of money and search that uses Kiyotaki and Wright (1989) as a paradigm rests on the random matching assumption in an economy with at least three kinds of individuals, a very expensive barter cost, to justify the emergence of a medium of exchange. In this case, the cost of bartering is an increasing function of population. Intuitively, it may be much less timeconsuming to transport the merchandize from one to the next door than randomly find somebody in particular in a population of, say, 50 individuals.

Suppose now that the technological conditions already exist, so inequality (4) holds, and consider either Figures 3 or 4. We will use next some ad hoc dynamics, as suggested by Fujita, Krugman and Venables (1999, chapter 1), to study how a redistribute barter economy can emerge out of an unstructured barter system ${ }^{1}$. If population is small, say less than $L^{*}$, producers will be better off if they undertake the shopping trip by themselves instead of dealing with a costly intermediary, so a spatial general equilibrium will be set in a PBE. Now, let population $(L)$ increases a bit and holds it there; if $L$ is still less than $L^{*}$, a new equilibrium in a PBE will be set, but once population surpasses $L^{*}$, some individuals will soon realize that they will be better off were they become intermediaries. Producers will also realize that they obtain a higher utility if they deal with a centralized merchant, and a RBE emerges.

\section{CONCLUSION}

The emergence of centralized merchants represents one step in the specialization process that allows increasing the advantages of trade. This is possible because centralized merchants allow producers to save time in barter activities and increase their production. Consequently, individuals obtain a higher level of consumption and utility.

\footnotetext{
${ }^{1}$ Anas (1992) and Fujita et. al. (1999, chapter 10) use similar ad hoc dynamics to study the emergence of new cities. Fujita et. al. (1999, chapter 1) provide an extensive discussion of the use of ad hoc dynamics to study the effects of population growth in the new economic geography and the modern evolutionary game theory.
} 
Note also that the improvement in the barter system caused by the emergence of centralized merchants allows increasing the efficiency of the economy and individuals' utility without needing to adopt a medium of exchange. Moreover, this occurs as a consequence of individual's rational decisions. However, some technological conditions and population size are necessary before this process takes hold. On the other hand, an improvement in the transport technology allows growth in both the number of goods produced and individuals' utility.

In the RBE, the economy can grow beyond the PBE limitations. The city size in the RBE now has only the restrictions imposed by the transport technology, which eventually makes impossible to produce beyond a certain radius. Yet, the limitation due to barter activities disappears since only a fixed and negligible amount of time is consumed in interacting with a merchant. This makes possible to lower unit costs.

Another interesting inquiry refers to the incentives to create a new city. Since equilibrium utility never falls to zero as population grow, this incentive does not exist, and the decrease in utility in the PBE is overcome by adopting a different barter system. The causes for the birth of new cities have different motives, as those mentioned in Anas (1992).

The model also provides an explanation to some primitive economies like the Peruvian Incas and the Egyptians during the Old Kingdom, which kept bartering and never adopted a medium of exchange [see Renfrew and Bahn (1996)].

Finally, one of the main features of ancient RBEs was the existence of a strong centralized authority that organized the redistribution of goods. In our model, this can be interpreted as follows. The government sets the market center in a central place. Yet, instead of limiting its activity to facilitate the redistribution of the merchandize evenly among merchants and let merchants work independently, it directly plays the role of the centralized merchant and hires the necessary number of individuals to do the merchants' job; $F$ is the number of merchants 
that the government should hire and $\xi$ is the revenue that the government receives for its services, that is, taxes. Notice that if the government decided to increase its revenue excessively and, for example, to hire too many merchants, taxes will also increase excessively, individuals' utility may decrease noticeable, and the whole system may fall. This was, perhaps, as argued by Einzig (1966, pg. 194), the main reason for the downfall of Egypt after the IV ${ }^{\text {th }}$ Dynasty.

\section{REFERENCES}

Anas, A. (1992). "On the Birth and Growth of Cities: Laissez-faire and Planning Compared." Regional Science and Urban Economics. 22, pg. 243-258. North Holland.

Brundage, Burr C. (1985). "Lords of Cuzco: A History and Description of the Inca People in Their Final Days." Second Edition. Oklahoma.

Clower, R. W. (1995). “On the Origins of Monetary Exchange.” Economic Inquiry, Vol. 33 October, 525-36.

Clower, R. W. and P. Howitt (2000). “The Emergence of Economic Organization.” Journal of Economic Behavior and Organization, 41(1), January, pages 55-84.

Davies, G. (1995). “A History of Money. From Ancient Times to the Present Day." University of Wales Press. Cardiff, UK.

Ederer, R. J. (1964). “The Evolution of Money.” Public Affairs Press. (1964).

Einzig, P. (1966). "Primitive Money. In its Ethnological, Historical and Economic Aspects." Pergamon Press. Second Edition.

Fujita, M., P. Krugman and A. J. Venables (1999). “The Spatial Economy. Cities, Regions and International Trade.” The MIT Press, Cambridge, Massachusetts.

Hicks, J. E. (1969). “A Theory of Economic History.” Clarendon Press. Oxford.

Hicks, J. E. (1989). “A Market Theory of Money.” Clarendon Press, Oxford. 
Kiyotaki, N. and R. Wright (1989). “On Money as a Medium of Exchange. "Journal of Political Economy, vol. 9, no. 41.

Polanyi, K. (1957). "The Economy as Instituted Process." in Trade and Market in the Early Empires Economies in History and Theory, Chapter XIII, edited by K. Polanyi, C. M. Arensberg and H. W. Pearson. The Free Press, NY. Collier-Macmillan Ltd, London.

Renfrew, C. and P. Bahn (1996). “Archeology. Theories Methods and Practice." Thames and Hudson Ltd. Second Edition, London.

Romer, John (1990). “Ancient Lives: Daily Life in Egypt of the Pharaohs”. Holt.

Samuelson, P. A. (1952). "The Transfer Problem and the transport Cost: The Terms of Trade when Impediments are Absent.” Economic Journal 62: 278-304.

von Thünen, J. H. (1826). "Der Isolierte Staat in Beziehung auf Landtschaft und Nationalökonomie.” Hamburg (English translation by C. M. Wartenberg, von Thünen's Isolated State, Oxford: Pergamon Press, 1966).

\section{APPENDIX A:}

First, let's define

$$
Z_{1}=(1-\gamma L)^{2-1 / \sigma}-(1-(\tau+\gamma) L)^{2-1 / \sigma}
$$

Taking derivatives respect to $L$, we have

$$
\begin{aligned}
& \frac{d Z_{1}}{d L}=\frac{2 \sigma-1}{\sigma}\left[(\tau+\gamma)(1-(\tau+\gamma) L)^{1-1 / \sigma}-\gamma(1-\gamma L)^{1-1 / \sigma}\right] \\
& \frac{d^{2} Z_{1}}{d L^{2}}=\frac{(2 \sigma-1)(\sigma-1)}{\sigma^{2}}\left[\gamma^{2}(1-\gamma L)^{-1 / \sigma}-(\tau+\gamma)^{2}(1-(\tau+\gamma) L)^{-1 / \sigma}\right] .
\end{aligned}
$$


Notice that $Z_{1}$ ' tends to $\tau(2 \sigma-1) / \sigma$ as $L$ tends to zero. Let's denote $M=\left(1+\frac{\tau}{\gamma}\right)^{\sigma /(\sigma-1)}$. Applying some tedious algebra, we find that $Z_{1}$ is positive and concave for any possible population size, and reaches a maximum at

$$
L^{*}=\frac{M-1}{M(\tau+\gamma)-\gamma}
$$

which less than that $1 /(\tau+\gamma)$, the maximum possible population. Now, we can proceed to prove the theorem. Since $U_{p b e}$ can be rewritten as $U_{p b e}(L)=\frac{A_{0}}{L} Z_{1}^{\sigma /(\sigma-1)}$, applying the L'Hopital rule, it can be easily verified that $U_{p b e}$ tends to zero as $L$ tends to zero. Also, by simple substitution, we have that $U_{p b e}=A_{0} \tau^{1+\sigma /(\sigma-1)}(\tau+\gamma)^{2+\sigma /(\sigma-1)}$ when $L=1 /(\tau+\gamma)$. This proves $(i)$ and $(i i)$.

Taking the first derivative, we find

$$
\frac{d U_{p b e}(L)}{d L}=A_{0}\left(\frac{\sigma}{\sigma-1} \frac{Z_{1}^{1 /(\sigma-1)}}{L} \frac{d Z_{1}}{d L}-\frac{Z_{1}^{\sigma /(\sigma-1)}}{L^{2}}\right)
$$

Also, applying the L'Hopital rule to take the limit as $L$ tends to zero, we find that the slope of $U_{p b e}$ tends to zero if $\sigma<2(\rho<0.5)$, tends to infinity if $\sigma>2(\rho>0.5)$, and tends to $Z_{1}$ ' $(0)$ if $\sigma=2$ $(\rho=0.5)$. This proves part (iii).

From the first derivative, notice that $d Z_{1}(0) / d L$ is positive if and only if

$$
\frac{d U_{p b e}(L)}{d L}=A_{0}\left(\frac{\sigma}{\sigma-1} \frac{Z_{1}^{1 /(\sigma-1)}}{L} \frac{d Z_{1}}{d L}-\frac{Z_{1}^{\sigma /(\sigma-1)}}{L^{2}}\right)
$$

This expression is positive (negative) if $d Z_{1}(L) / d L$ is greater (less) than $\frac{(\sigma-1)}{\sigma} \frac{Z_{1}(L)}{L}$, which is always positive. Also, it can be easily checked that, as $L$ tends to zero, $d Z_{1}(L) / d L$ is greater than $\frac{(\sigma-1)}{\sigma} \frac{Z_{1}(L)}{L}$, and as $L$ tends to the maximum population $1 /(\tau+\gamma), d Z_{1}(L) / d L$ is less than 
$\frac{(\sigma-1)}{\sigma} \frac{Z_{1}(L)}{L}$. Since $Z_{1}$ and $d Z_{1} / d L$ are both continuously differentiable in the interval $(0$,

$1 /(\tau+\gamma)$ ), from the Brower fix point theorem we have that there exist $L_{P}$ between 0 and $1 /(\tau+\gamma)$ such that $d U_{p b e}\left(L_{P}\right) / d L=0$.

Q.E.D.

\section{APPENDIX B:}

First, let's define

$$
Z_{2}=1-(1-\tau G(L))^{2-1 / \sigma}
$$

Taking derivatives respect to $L$, we have

$$
\frac{d Z_{2}}{d L}=\frac{\tau(2 \sigma-1)}{\sigma}(1-\tau G(L))^{1-1 / \sigma} G^{\prime}(L) .
$$

Notice that, as $L$ tends to zero, $d Z_{2} / d L$ tends to $(2 \sigma-1) K / x \sigma \eta$. Since $G(L)$ is always increasing, $d Z_{2} / d L$ is always positive, so $Z_{2}$ is always an increasing function with respect to $L$.

Now, we can proceed to prove the theorem. Notice that $U_{b e m}(L)=\frac{A_{0}}{L} Z_{2}^{\sigma /(\sigma-1)}$, applying the L'Hopital rule, we can check that $U_{r b e}$ tends to zero as $L$ tends to zero. This proves (i). Also, by substitution it can be easily checked that (ii) and (iii) hold.

Taking the first derivative with respect to $L$, we find that $d U_{r b e} / d L$ is positive if

$$
\frac{\tau(2 \sigma-1)}{\sigma-1} G^{\prime}(L) L>0>(1-\tau G(L))^{1 / \sigma-1}-(1-\tau G(L))
$$

since $\sigma>1$ and $\tau G(L)<1$. Thus, $U_{r b e}$ is always increasing. This proves the theorem.

Q.E.D. 


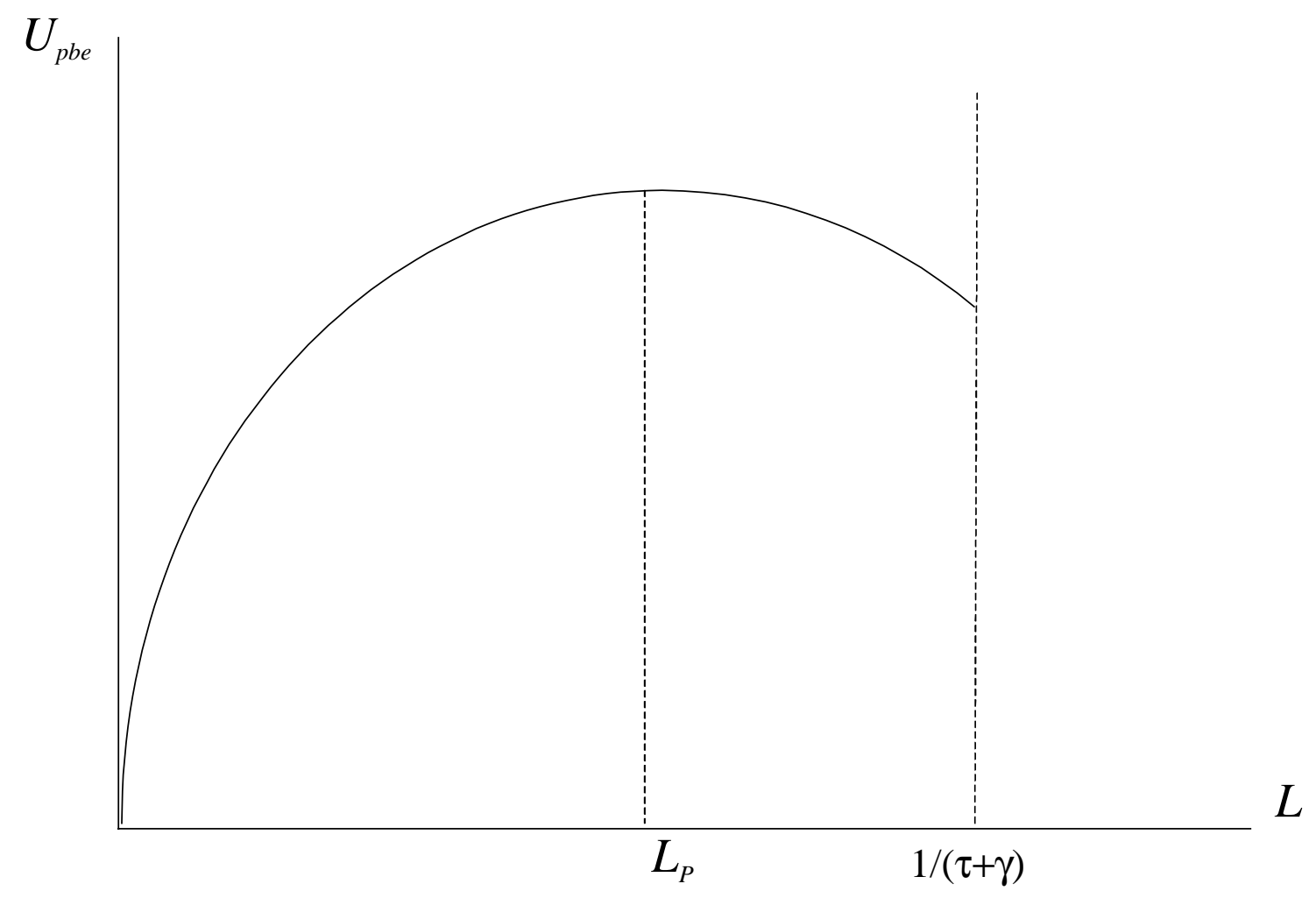

FIGURE ONE

The Utility Function in the Primitive Barter Economy 


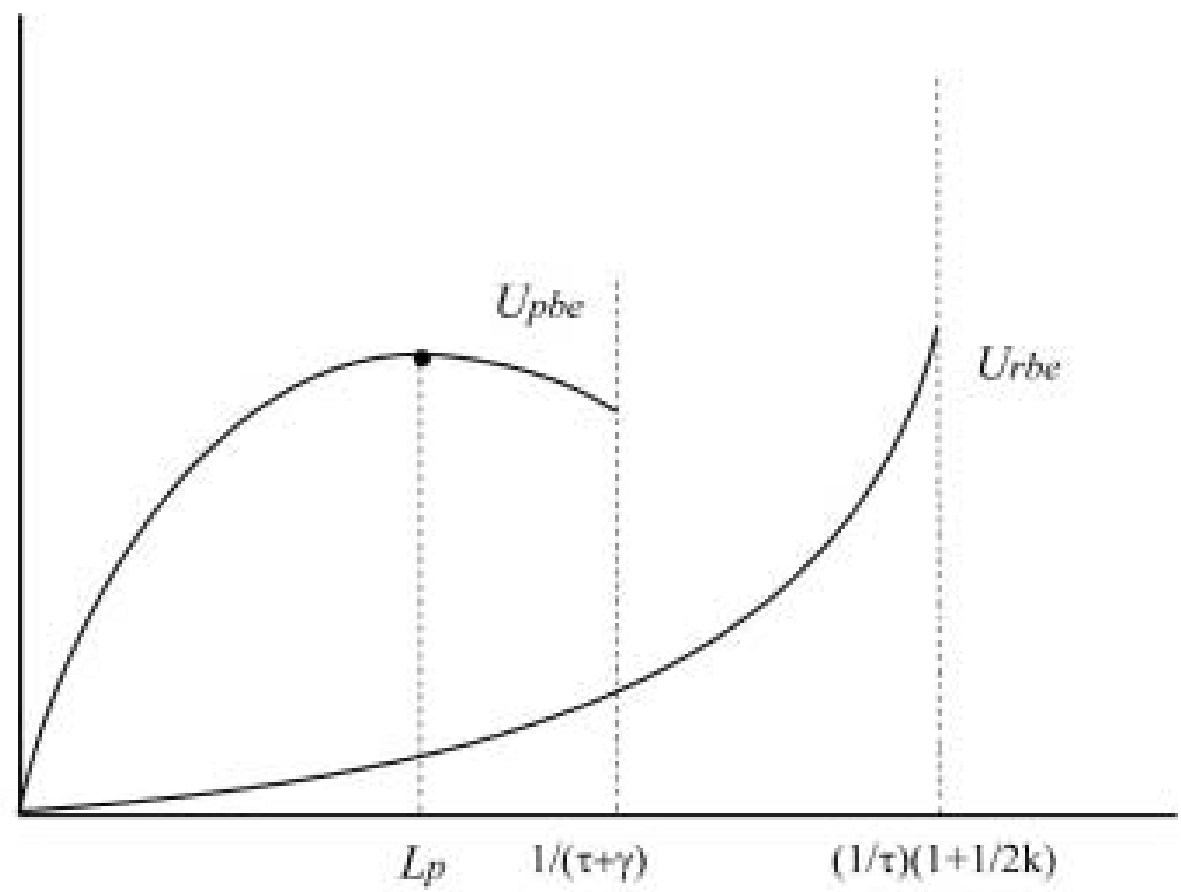

FIGURE TWO

THE PBE Always Dominates the RBE 


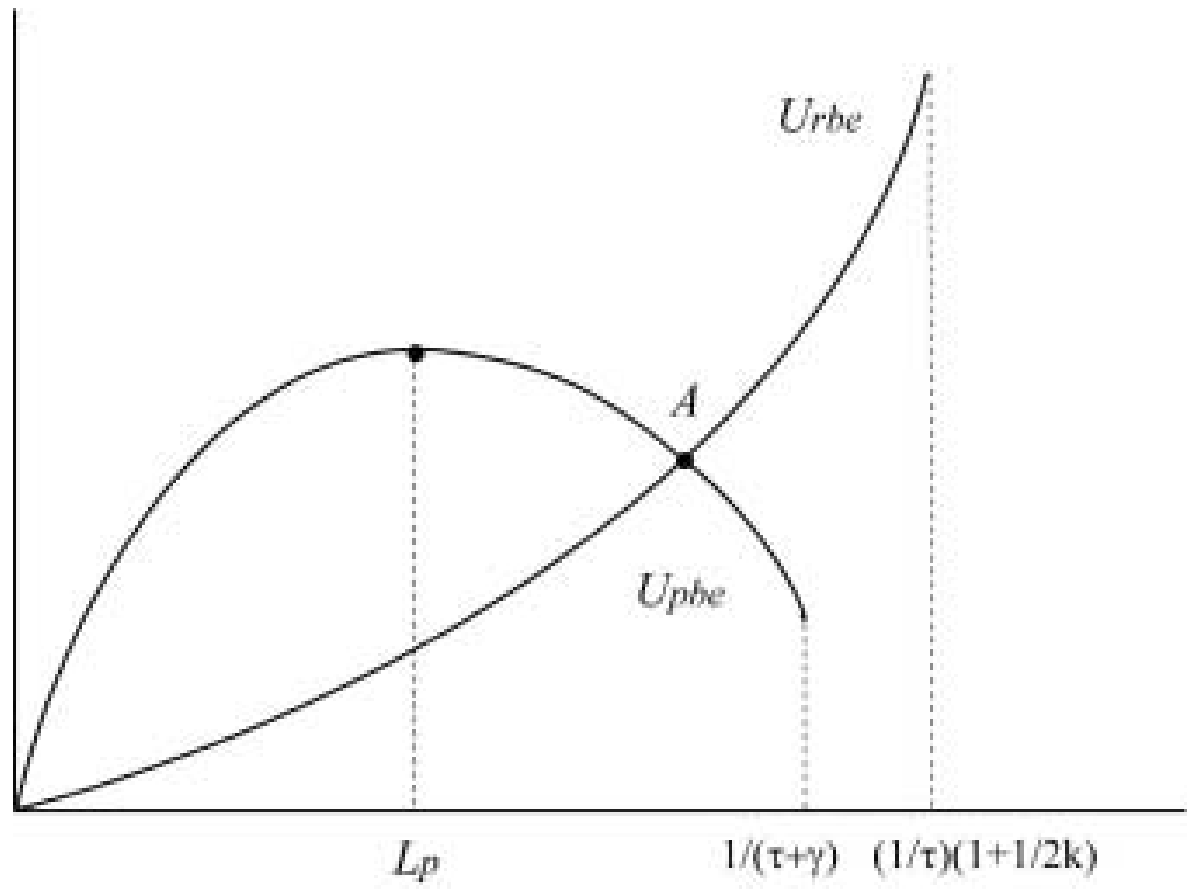

FIGURE THREE

RBE Dominates the PBE After It Reaches the Maximum 


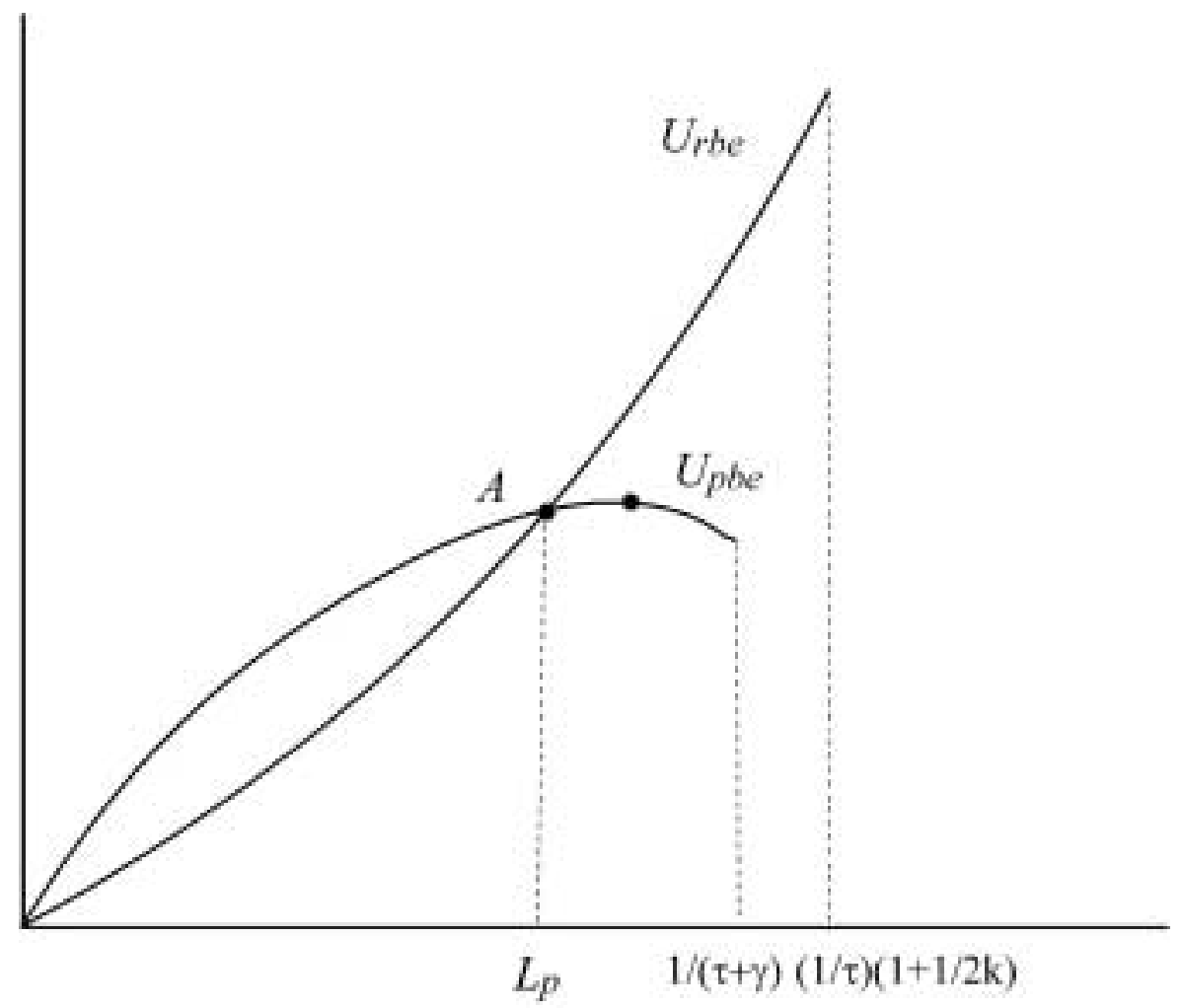

FIGURE FOUR

RBE Dominates the PBE Before It Reaches the Maximum 